\title{
Effectiveness of seasonal influenza vaccine for adults and children in preventing laboratory-confirmed influenza in primary care in the United Kingdom: 2015/16 end-of- season results
}

R Pebody ${ }^{1}$, F Warburton ${ }^{1}$, J Ellis ${ }^{1}$, N Andrews ${ }^{1}$, A Potts ${ }^{2}$, S Cottrell ${ }^{3}$, J Johnston ${ }^{4}$, A Reynolds ${ }^{2}$, R Gunson ${ }^{5}$, C Thompson 1, M Galiano ${ }^{1}$, C Robertson ${ }^{6}$, R Byford ${ }^{7}$, N Gallagher ${ }^{4}$, M Sinnathamby ${ }^{1}$, I Yonova ${ }^{7}{ }^{8}$, S Pathirannehelage ${ }^{7}$, M Donati ${ }^{1}$, C Moore $^{3}, \mathrm{~S}$ de Lusignan ${ }^{78}$, J McMenamin ${ }^{2}$, M Zambon ${ }^{1}$

1. Public Health England, London, United Kingdom

2. Health Protection Scotland, Glasgow, United Kingdom

3. Public Health Wales, Cardiff, United Kingdom

4. Public Health Agency Northern Ireland, Belfast, United Kingdom

5. West of Scotland Specialist Virology Centre, Glasgow, United Kingdom

6. University of Strathclyde, Glasgow, United Kingdom

7. University of Surrey, Guildford, United Kingdom

8. Royal College of General Practitioners, London, United Kingdom

Correspondence: Richard Pebody (richard.pebody@phe.gov.uk)

Citation style for this article:

Pebody R, Warburton F, Ellis J, Andrews N, Potts A, Cottrell S, Johnston J, Reynolds A, Gunson R, Thompson C, Galiano M, Robertson C, Byford R, Gallagher N, Sinnathamby M, Yonova I, Pathirannehelage S, Donati M, Moore C, de Lusignan S, McMenamin J, Zambon M. Effectiveness of seasonal influenza vaccine for adults and children in preventing laboratory-confirmed influenza in primary care in the United Kingdom: 2015/16 end-of-season results. Euro Surveill. 2016;21(38):pii=30348. DOI: http://dx.doi.org/10.2807/1560-7917.ES.2016.21.38.30348

The United Kingdom (UK) is in the third season of introducing universal paediatric influenza vaccination with a quadrivalent live attenuated influenza vaccine (LAIV). The 2015/16 season in the UK was initially dominated by influenza $\mathrm{A}\left(\mathrm{H}_{1} \mathrm{~N}_{1}\right)$ pdmog and then influenza of B/Victoria lineage, not contained in that season's adult trivalent inactivated influenza vaccine (IIV). Overall adjusted end-of-season vaccine effectiveness (VE) was $52.4 \%$ (95\% confidence interval $(\mathrm{Cl})$ : 41.0-61.6) against influenza-confirmed primary care consultation, $54.5 \%$ ( $95 \% \mathrm{Cl}$ : 41.6-64.5) against influenza $A\left(\mathrm{H}_{1} \mathrm{~N}_{1}\right)$ pdmog and $54.2 \%(95 \% \mathrm{Cl}$ : 33.1-68.6) against influenza $B$. In 2-17 year-olds, adjusted VE for LAIV was $57.6 \%$ (95\% Cl: 25.1 to 76.0 ) against any influenza, 81.4\% (95\% Cl: 39.6-94.3) against influenza $\mathrm{B}$ and $41.5 \%(95 \% \mathrm{Cl}:-8.5$ to 68.5$)$ against influenza $A\left(\mathrm{H}_{1} \mathrm{~N}_{1}\right)$ pdmo9. These estimates demonstrate moderate to good levels of protection, particularly against influenza $B$ in children, but relatively less against influenza $A\left(\mathrm{H}_{1} \mathrm{~N}_{1}\right)$ pdmo9. Despite lineage mismatch in the trivalent IIV, adults younger than 65 years were still protected against influenza $B$. These results provide reassurance for the UK to continue its influenza immunisation programme planned for 2016/17.

\section{Introduction}

The United Kingdom (UK) has had a long-standing selective inactivated influenza vaccination programme targeted at individuals at higher risk of severe disease such as the elderly, those with an underlying clinical risk condition and pregnant women. Following recommendations from the Joint Committee of Vaccination and Immunisation (JCVI) in 2012, the decision was taken for a phased introduction of a newly licensed live attenuated influenza vaccine (LAIV), ultimately offered LAIV in each season to all healthy children aged two to 16 years [1]. 2015/16 is the third season of the introduction of this new influenza vaccination programme; all healthy children aged two to four years and in school years 1 and 2 were offered a single dose of LAIV [2]. In Northern Ireland and Scotland and in selected pilot areas in England, all other older children of primary school age were also offered LAIV in 2015/16. Children aged two to 17 years in a clinical risk group were also offered LAIV, while children with a risk factor, in whom LAIV is contraindicated, were offered quadrivalent inactivated influenza vaccine (IIV). All children in a clinical risk group aged six to 23 months were offered IIV. The United States Centers for Disease Control and Prevention (US CDC) recently reported the observation that LAIV did not provide protection in children against circulating influenza strains in North America in the 2015/16 season [3]. This raised a question about the effectiveness of LAIV in children in the UK.

In the UK, the 2015/16 season started late, peaking in week 11 of 2016, with circulation initially dominated by influenza $\mathrm{A}\left(\mathrm{H}_{1} \mathrm{~N}_{1}\right)$ pdmog viruses. Impact mainly fell on younger adults resulting in large numbers of hospitalisations and admissions to intensive care units (ICU) [4]. 


\section{FIGURE 1}

Specimen inclusion and exclusion criteria, end-of-season 2015/16 influenza vaccine effectiveness evaluation, United Kingdom, 1 October 2015-1 May 2016 (n = 5,811)

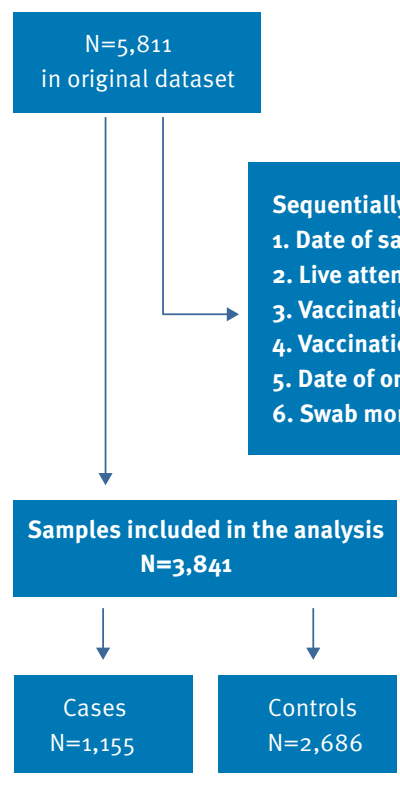

Genetically, the haemagglutinin $(\mathrm{HA})$ genes of $A\left(\mathrm{H}_{1} \mathrm{~N}_{1}\right)$ pdmog viruses all belonged in subgroup 6B, the predominant clade circulating in the 2014/15 season. The later stages of the $2015 / 16$ season were dominated by influenza B circulation, with the majority of viruses antigenically similar to B/Brisbane/60/2008, the influenza $\mathrm{B} /$ Victoria lineage component included in the 2015/16 northern hemisphere quadrivalent vaccine but not in the trivalent vaccine [4]. This raised questions about the protection provided by the 2015/16 trivalent vaccine, the main influenza vaccine offered to adults, and about the potential added value of switching to quadrivalent vaccine as the main vaccine of choice.

Following the mid-2015/16 season report of influenza vaccination effectiveness (VE) [5], this article presents the end-of-season estimates of influenza VE using well established systems across the four countries of the UK $[6,7]$. The aims of the investigation were to measure VE against laboratory-confirmed influenza by type, subtype and clade/lineage, and to determine the effectiveness of the vaccine in children two to 17 years of age according to type of vaccine, particularly in relation to LAIV, but also IIV. In addition, we estimated the effectiveness of both LAIV and IIV in children two to 17 years of age over the three seasons since the UK introduced the LAIV programme.

\section{Methods}

\section{Study population and period}

The test-negative case-control (TNCC) design was used to estimate VE. The study was undertaken in five sentinel general practice surveillance networks across the UK, details of which have been outlined previously [7]. The surveillance schemes were: Royal College of General Practitioners (RCGP), Research and Surveillance Centre (RSC), Specialist Microbiology Network (SMN) England and Wales, Northern Ireland and Scotland.

The main study took place from 1 October 2015 until 1 May 2016. The study population were patients presenting to their general practitioner (GP) during the study period with an acute influenza-like illness (ILI), who the physician consented verbally to be swabbed during the consultation. A patient with ILI was defined as an individual presenting in primary care with an acute respiratory illness with physician-diagnosed fever or complaint of feverishness. GPs were asked to swab a random sample of cases up to a total of 10 per week in any one practice. Cases were patients who tested positive for influenza $A$ or $B$ virus by real-time $P C R$. Controls were patients with the same symptoms who tested negative for influenza A and B. Further details of the laboratory methods are provided below.

During the consultation, the GP completed a standard questionnaire. It collected demographic, clinical and epidemiological information from patients including potential confounders such as sex, date of birth, underlying clinical risk factors, date of onset of ILI, date of sample collection (recommended within seven days of onset) and influenza vaccination history for the 2015/16 season including date of vaccination and route of administration (intranasal/injection). In England, residence in an area where a primary school LAIV immunisation programme took place was also recorded.

A further specific sub-analysis was undertaken among children two to 17 years of age for the period 1 October 2013 until 1 May 2016. This covered the period since the introduction of LAIV in the UK. All aspects of data collection, testing and analysis were comparable over this period and are as described above.

\section{Laboratory methods}

Sentinel GP surveillance networks sent the respiratory samples to the national laboratories as previously outlined [7]. Laboratory confirmation was made using comparable real-time $P C R$ methods able to detect circulating influenza $A$ and $B$ viruses $[8,9]$. Positive samples were sent to the reference laboratories for genetic characterisation. Isolation of influenza viruses was tried from all PCR-positive samples using MadinDarby canine kidney epithelial (MDCK) cells or MDCK cells containing the CDNA of human 2,6-sialtransferase (SIAT1) cells as described previously $[10,11]$.

Antigenic characterisation was only undertaken at the PHE reference laboratory. Post-infection ferret antisera were used in haemagglutination inhibition ( $\mathrm{HI}$ ) assays with turkey red blood cells to antigenically characterise influenza $A\left(\mathrm{H}_{1} \mathrm{~N}_{1}\right)$ pdmog and influenza $B$ virus isolates with a haemagglutination titre $\geq 40$ [12]. Reference virus strains used for $\mathrm{HI}$ assays for $\mathrm{A}\left(\mathrm{H}_{1} \mathrm{~N}_{1}\right)$ pdmog 


\section{FIGURE 2}

Phylogenetic tree of the haemagglutinin genes of sentinel influenza B isolates, United Kingdom, October 2015-May 2016 $(\mathrm{n}=324)$

B/Victoria lineage

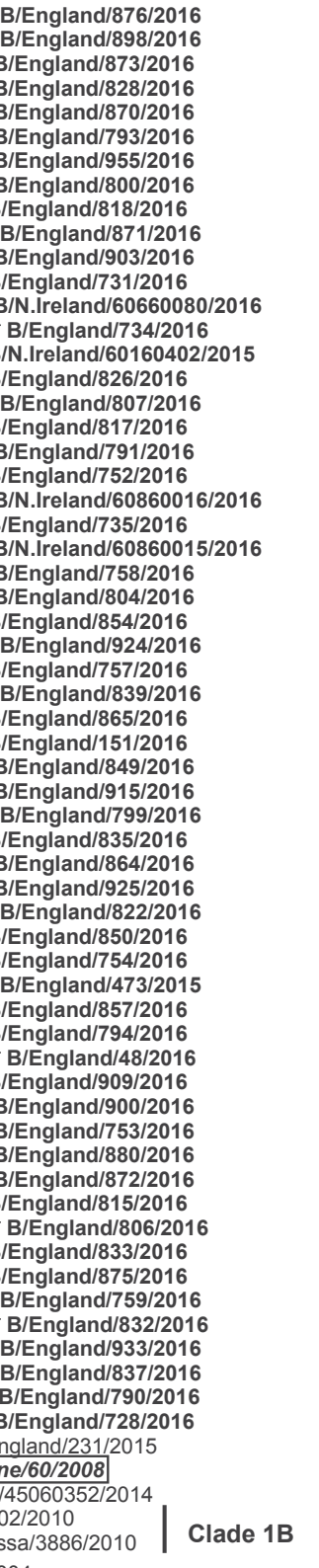

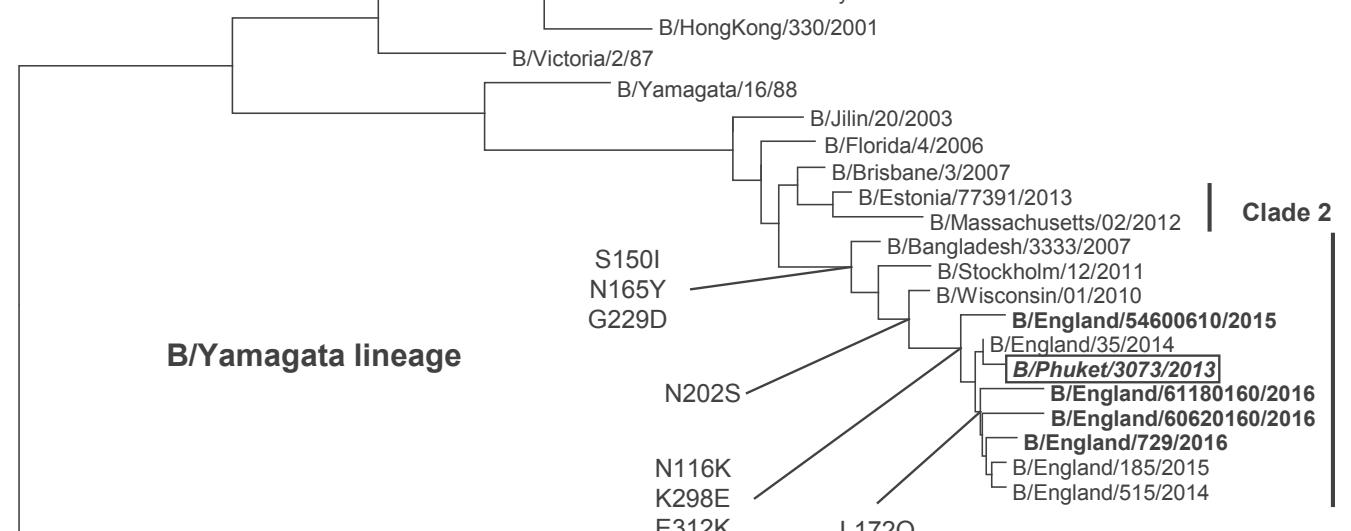


TABLE 1

Influenza B haemagglutinin sequences obtained from GISAID used in the phylogenetic analysis

\begin{tabular}{|c|c|c|c|c|c|}
\hline Influenza virus isolate & $\begin{array}{l}\text { Segment ID/ } \\
\text { Accession } \\
\text { number }\end{array}$ & Country & $\begin{array}{l}\text { Collection } \\
\text { date (year- } \\
\text { month-day) }\end{array}$ & Originating laboratory & Submitting laboratory \\
\hline B/Brisbane/3/2007 & EPI154537 & Australia & 2007-Jan-01 & $\begin{array}{l}\text { Queensland Health } \\
\text { Scientific Services, } \\
\text { Queensland, Australia }\end{array}$ & $\begin{array}{l}\text { WHO Collaborating } \\
\text { Centre for Reference and } \\
\text { Research on Influenza, } \\
\text { Victoria, Australia }\end{array}$ \\
\hline B/Stockholm/12/2011 & $\mathrm{EPI} 346827$ & Sweden & 2011-Mar-28 & $\begin{array}{l}\text { Swedish Institute for } \\
\text { Infectious Disease } \\
\text { Control, Solna, Sweden }\end{array}$ & $\begin{array}{c}\text { National Institute for } \\
\text { Medical Research, London, } \\
\text { UK } \\
\end{array}$ \\
\hline B/England/515/2014 & EPI555201 & $\begin{array}{l}\text { United } \\
\text { Kingdom }\end{array}$ & 2014-Oct-22 & $\begin{array}{l}\text { Public Health England, } \\
\text { London, UK }\end{array}$ & $\begin{array}{c}\text { National Institute for } \\
\text { Medical Research, London, } \\
\text { UK }\end{array}$ \\
\hline B/Estonia/77391/2013 & $\mathrm{EPI} 467120$ & Estonia & 2013-Apr-08 & $\begin{array}{c}\text { Health Protection } \\
\text { Inspectorate, Tallin, } \\
\text { Estonia } \\
\end{array}$ & $\begin{array}{c}\text { National Institute for } \\
\text { Medical Research, London, } \\
\text { UK }\end{array}$ \\
\hline $\mathrm{B} /$ dessa/3886/2010 & $\mathrm{EPI} 271913$ & Ukraine & 2010-Mar-19 & $\begin{array}{l}\text { Ministry of Health of } \\
\text { Ukraine, Kiev, Ukraine }\end{array}$ & $\begin{array}{c}\text { National Institute for } \\
\text { Medical Research, London, } \\
\text { UK }\end{array}$ \\
\hline B/Phuket/3073/2013 & $\mathrm{EPI}_{540675}$ & Australia & 2013-Nov-21 & $\begin{array}{c}\text { WHO Collaborating } \\
\text { Centre for Reference and } \\
\text { Research on Influenza, } \\
\text { Victoria, Australia }\end{array}$ & $\begin{array}{c}\text { National Institute for } \\
\text { Medical Research, London, } \\
\text { UK }\end{array}$ \\
\hline $\mathrm{B} /$ Massachusetts/02/2012 & $\mathrm{EPI}_{438406}$ & United States & 2012-Jan-01 & $\begin{array}{c}\text { New York Medical } \\
\text { College, New York, US }\end{array}$ & $\begin{array}{c}\text { Centers for Disease } \\
\text { Control and Prevention, } \\
\text { Atlanta, US }\end{array}$ \\
\hline B/Wisconsin/01/2010 & $\mathrm{EPI} 271545$ & United States & $2010-$ Feb-20 & $\begin{array}{c}\text { Wisconsin State } \\
\text { Laboratory of Hygiene, } \\
\text { Madison, US }\end{array}$ & $\begin{array}{c}\text { Centers for Disease } \\
\text { Control and Prevention, } \\
\text { Atlanta, US }\end{array}$ \\
\hline B/Hawaii/02/2010 & $\mathrm{EPI} 271558$ & United States & 2010-Mar-25 & $\begin{array}{c}\text { State of Hawaii } \\
\text { Department of Health, } \\
\text { Pearl City, US }\end{array}$ & $\begin{array}{c}\text { Centers for Disease } \\
\text { Control and Prevention, } \\
\text { Atlanta, US } \\
\end{array}$ \\
\hline $\mathrm{B} /$ Brisbane $/ 60 / 2008$ & EPI172555 & Australia & 2008-Aug-04 & & $\begin{array}{c}\text { Centers for Disease } \\
\text { Control and Prevention, } \\
\text { Atlanta, US }\end{array}$ \\
\hline B/Florida/4/2006 & $\mathrm{EPI}_{134356}$ & United States & 2006-Nov-01 & & $\begin{array}{c}\text { Centers for Disease } \\
\text { Control and Prevention, } \\
\text { Atlanta, US }\end{array}$ \\
\hline B/Bangladesh/3333/2007 & $\mathrm{EPI} 156050$ & Bangladesh & 2007-Aug-18 & & $\begin{array}{c}\text { Centers for Disease } \\
\text { Control and Prevention, } \\
\text { Atlanta, US }\end{array}$ \\
\hline
\end{tabular}

GISAID: Global Initiative on Sharing All Influenza Data; UK: United Kingdom; US: United States; WHO: World Health Organization.

viruses included $\mathrm{A} /$ California/7/2009 (vaccine strain) grown in embryonated chicken eggs and other $\mathrm{A}\left(\mathrm{H}_{1} \mathrm{~N}_{1}\right)$ pdmog England strains grown in embryonated chicken eggs or tissue culture cells. Reference virus strains used for $\mathrm{HI}$ assays for influenza $\mathrm{B}$ viruses included B/ Phuket/3073/2013 (trivalent and quadrivalent vaccine strain) and B/Brisbane/6o/2008 (quadrivalent vaccine strain) together with a panel of other egg- and tissue culture-grown influenza $B$ viruses from both the $B /$ Yamagata/16/88-lineage and the $B / V i c t o r i a / 2 / 87$ lineage. The fold difference between the homologous $\mathrm{HI}$ titre for the corresponding vaccine strain and the $\mathrm{HI}$ titre for each clinical isolate was calculated to determine antigenic similarity of clinical isolates to the vaccine strain.
Nucleotide sequencing of the haemagglutinin (HA) gene was undertaken (primer sequences available on request) for a subset of influenza $A\left(\mathrm{H}_{1} \mathrm{~N}_{1}\right)$ pdmog and $B$ viruses selected to be representative of the range of the patients' age, date of sample collection, geographical location and, if performed, antigenic characterisation of the virus isolate, and phylogenetic trees were constructed with a neighbour-joining algorithm available in the Mega 6 software (http://www.megasoftware.net) [13]. The $A\left(\mathrm{H}_{1} \mathrm{~N}_{1}\right)$ pdmog results have been previously presented [5]. HA sequences from reference strains used in the phylogenetic analysis for influenza B in this paper were obtained from GenBank: B/Malaysia/2506/2004 (CYo38287), B/Jilin/20/2003 (CYo33828), B/Yamagata/16/88 (CYo18765), B/ Victoria/2/87 (M58428), B/HongKong/330/2001 (AF532549) and from the EpiFlu database of the Global 
Initiative on Sharing All Influenza Data (GISAID) (Table 1).

\section{Statistical methods}

Patients were defined as vaccinated if they had received the 2015/16 seasonal vaccine at least 14 days before first onset of ILI. Patients were excluded if they were vaccinated less than 14 days before symptom onset. If vaccinated, but date of vaccination was unknown, the median date of vaccination of those with known dates was taken instead. Patients with date of onset not known or onset more than seven days before swabbing were also excluded. A similar approach was used to undertake a pooled analysis for the 2013/14, 2014/15 and 2015/16 seasons.

The odds ratios (OR) obtained from multivariable logistic regression models were used to calculate VE with influenza laboratory results as the outcome and influenza vaccination status as the linear predictor. Influenza $A\left(\mathrm{H}_{1} \mathrm{~N}_{1}\right)$ pdmog- and influenza B-specific VE was also calculated. Samples positive for other subtypes were excluded as the numbers were too small, except for the three-season pooled analysis, which also included influenza $\mathrm{A}\left(\mathrm{H}_{3} \mathrm{~N}_{2}\right)$. The adjusted estimates were set based on past seasons as age (age groups: $0-4,5-17,18-44,45-64, \geq 65$ years), month of sample collection, residence in area where a primary school programme was in place, sex and surveillance scheme. We also explored whether being in a risk group for whom vaccination is recommended provided any evidence of confounding. For the three-year pooled analysis, year was also included in the model. All statistical analyses were carried out in Stata version 13 (StataCorp, College Station, Texas).

The HA sequences from England obtained in this study, which were also used in the phylogenetic analysis, were deposited in GISAID under the following accession numbers: EPI679258, EPI811543, EPI811551, EPI811554, EPI811562, EPI811570, EPI811578, EPI811586, EPI811594, EPI811598, EPI811606, EPI811614, EPI811622, EPI811626, EPI811629, EPI811637, EPI811645, EPI811648, EPI811656, EPI811664, EPI811671, EPI811675, EPI811683, EPI811691, EPI811699, EPI811707, EPI811715, EPI811723, EPI811726, EPI811734, EPI811742, EPI811750, EPI811758, EPI811766, EPI811774, EPI811782, EPI811788, EPI811796, EPI811799, EPI811807, EPI811815, EPI811823, EPI811831, EPI811839, EPI811842, EPI811845, EPI811853, EPI811856, EPI811864, EPI811868, EPI811876, EPI811884, EPI811891, EPI811894, EPI811898, EPI811906, EPI811909, EPI811915, EPI811916, EPI811924, EPI811932, EPI811940, EPI811944, EPI811952, EPI811958.

\section{Results}

Of the 5,811 swabbed individuals potentially eligible, 3,841 individuals were confirmed eligible and included in the study (Figure 1). The details of those included in the study are provided by swab result in Table 2, including those with missing data. There were a total of 2,686 controls, 351 (9.1\%) influenza B detections, $770 \mathrm{~A}\left(\mathrm{H}_{1} \mathrm{~N}_{1}\right)$ pdmog detections (20.0\%), 24 influenza $\mathrm{A}\left(\mathrm{H}_{3} \mathrm{~N}_{2}\right)$ detections (o.6\%) and 15 influenza A(untyped) detections (0.4\%). Four samples tested positive for both $\mathrm{A}\left(\mathrm{H}_{1} \mathrm{~N}_{1}\right)$ pdmog and influenza $B$ and one sample was positive for both $A\left(\mathrm{H}_{1} \mathrm{~N}_{1}\right) p d m o 9$ and $A\left(\mathrm{H}_{3} \mathrm{~N}_{2}\right)$. Positivity rates differed significantly by age group, sex, risk group, month, scheme, vaccination status and area of primary school programme in England (Table 2).

\section{Influenza $\mathrm{A}(\mathrm{H} 1 \mathrm{N1}) \mathrm{pdm} 09$ and $\mathrm{B}$ strain} characterisation from sentinel samples

Since week 40 in 2015, a total of 730 influenza viruses from this study have been characterised by the PHE Respiratory Virus Unit and the West of Scotland Virology Centre: 128 antigenically, 293 genetically and 309 through both methods. Only the PHE Respiratory Virus Unit undertook the antigenic analysis.

A total of 482 influenza $A\left(\mathrm{H}_{1} \mathrm{~N}_{1}\right)$ pdmog viruses were characterised. All belonged in the genetic subgroup $6 \mathrm{~B}$, which had been the predominant genetic subgroup in the $2014 / 15$ season. Some heterogeneity was seen in the HA of the current season's $A\left(\mathrm{H}_{1} \mathrm{~N}_{1}\right)$ pdmog viruses, with some newly emerging genetic subgroups: the HA genes of the majority (93\%) of $A\left(\mathrm{H}_{1} \mathrm{~N}_{1}\right)$ pdmog viruses fell into genetic cluster 6B.1, characterised by the amino acid changes $\mathrm{S} 84 \mathrm{~N}, \mathrm{~S}_{16} \mathrm{~N}$ (with gain of a potential glycosylation site) and $1216 \mathrm{~T}$, with a subset in this cluster having the substitution $A 215 \mathrm{G}$. Less than $6 \%$ of viruses fell into a second emerging cluster (6B.2) and had the amino acid substitutions $\mathrm{V}_{152} \mathrm{~T}, \mathrm{~V}_{173} \mathrm{I}$, E491G and D501E in the HA gene, or into a third minor cluster with substitutions N129D, R450K and E491G. A few viruses from this season did not have any of these changes or had only the substitution $\mathrm{S} 84 \mathrm{~N}$, and clustered with $\mathrm{A}\left(\mathrm{H}_{1} \mathrm{~N}_{1}\right)$ pdmog viruses from season 2014/15 (6B subgroup). A tree showing the phylogenetic relationships for the $\mathrm{A}\left(\mathrm{H}_{1} \mathrm{~N}_{1}\right)$ pdmog has already been published [5]. Of 123 $A\left(\mathrm{H}_{1} \mathrm{~N}_{1}\right)$ pdmog viruses isolated from sentinel samples between December 2015 and April 2016 and analysed by $\mathrm{HI}$ assay using an extended panel of ferret postinfection sera including a ferret post-infection antiserum to $A / C a l i f o r n i a / 7 / 2009$ (NIBSC, UK), 100\% were antigenically similar to the $A / C a l i f o r n i a / 7 / 2009$ northern hemisphere 2015/16 $\mathrm{A}\left(\mathrm{H}_{1} \mathrm{~N}_{1}\right)$ pdmog vaccine strain. Using this extended panel of ferret post-infection antisera, no antigenic low reactors to A/California/7/2009 antisera were observed.

A total of 324 influenza B viruses were characterised: more than $96 \%$ of them belonged to the $B /$ Victoria lineage in clade $1 \mathrm{~A}$, represented by $\mathrm{B} /$ Brisbane/60/2008 (the 2015/16 quadrivalent vaccine strain) (Figure 2). Viruses in this clade have N75K, N165K and S172P in their HA compared with the previous vaccine virus. Additional amino acid substitutions seen this season were I117V, N129D and V146I. A few (<3\%) UK 2015/16 $\mathrm{B} /$ Yamagata lineage viruses were detected, all belonging to genetic clade 3, with amino acid substitutions S15ol, N165Y and G229D relative to a previous vaccine 
TABLE 2

\begin{tabular}{|c|c|c|c|c|c|c|c|c|c|c|c|c|}
\hline & \multicolumn{2}{|c|}{ Controls } & \multicolumn{2}{|c|}{ Influenza Ba } & \multicolumn{2}{|c|}{$\begin{array}{l}\text { Influenza } \\
\mathrm{A}\left(\mathrm{H}_{1} \mathrm{~N}_{1}\right)^{\mathrm{a}}\end{array}$} & \multicolumn{2}{|c|}{$\begin{array}{c}\text { Influenza } \\
\mathrm{A}\left(\mathrm{H}_{3} \mathrm{~N}_{2}\right)\end{array}$} & \multicolumn{2}{|c|}{$\begin{array}{c}\text { Influenza } \\
\text { A(untyped) }\end{array}$} & Total $^{a}$ & $p$ value $^{b}$ \\
\hline \multicolumn{13}{|l|}{ Age group (years) } \\
\hline $0-4$ & 273 & 71.3 & 19 & 5.0 & 91 & 23.8 & 1 & 0.3 & 1 & 0.3 & 383 & \multirow{6}{*}{$<0.0001$} \\
\hline $5-17$ & 392 & 69.3 & 92 & 16.3 & 78 & 13.8 & 5 & 0.9 & 1 & 0.2 & 566 & \\
\hline $18-44$ & 1,022 & 65.9 & 170 & 11.0 & 348 & 22.4 & 7 & 0.5 & 5 & 0.3 & 1,551 & \\
\hline $45-64$ & 636 & 70.0 & 47 & 5.2 & 211 & 23.2 & 7 & 0.8 & 7 & 0.8 & 908 & \\
\hline$\geq 65$ & 346 & 84.6 & 19 & 4.6 & 39 & 9.5 & 4 & 1.0 & 1 & 0.2 & 409 & \\
\hline Missing & 17 & 70.8 & 4 & 16.7 & 3 & 12.5 & 0 & 0 & 0 & 0 & 24 & \\
\hline \multicolumn{13}{|l|}{ Sex } \\
\hline Female & 1,627 & 72.4 & 188 & 8.4 & 417 & 18.5 & 12 & 0.5 & 8 & 0.4 & 2,248 & \multirow{3}{*}{$<0.0001$} \\
\hline Male & 1,046 & 66.4 & 162 & 10.3 & 350 & 22.2 & 12 & 0.8 & 7 & 0.4 & 1,576 & \\
\hline Missing & 13 & 76.5 & 1 & 5.9 & 3 & 17.6 & 0 & 0 & 0 & 0 & 17 & \\
\hline \multicolumn{13}{|l|}{ Surveillance scheme } \\
\hline Northern Ireland & 76 & 49.0 & 22 & 14.2 & 51 & 32.9 & 0 & 0 & 6 & 3.9 & 155 & \multirow{5}{*}{$<0.0001$} \\
\hline RCGP & 1,148 & 64.0 & 179 & 10.0 & 449 & 25.0 & 19 & 1.1 & 0 & 0 & 1,793 & \\
\hline SMN & 138 & 67.0 & 12 & 5.8 & 50 & 24.3 & 1 & 0.5 & 5 & 2.4 & 206 & \\
\hline Scotland & 1,242 & 81.8 & 101 & 6.6 & 172 & 11.3 & 3 & 0.2 & 4 & 0.3 & 1,519 & \\
\hline Wales & 82 & 48.8 & 37 & 22.0 & 48 & 28.6 & 1 & 0.6 & 0 & 0 & 168 & \\
\hline \multicolumn{13}{|l|}{ Risk group } \\
\hline No & 1,794 & 66.5 & 276 & 10.2 & 607 & 22.5 & 14 & 0.5 & 9 & 0.3 & 2,697 & \multirow{3}{*}{$<0.0001$} \\
\hline Yes & 817 & 79.7 & 53 & 5.2 & 141 & 13.8 & 9 & 0.9 & 6 & 0.6 & 1,025 & \\
\hline Missing & 75 & 63.0 & 22 & 18.5 & 22 & 18.5 & 1 & 0.8 & 0 & 0 & 119 & \\
\hline \multicolumn{13}{|l|}{ Interval onset-sample (days) } \\
\hline $0-1$ & 292 & 67.6 & 41 & 9.5 & 95 & 22.0 & 2 & 0.5 & 2 & 0.5 & 432 & \multirow{3}{*}{$<0.0001$} \\
\hline $2-4$ & 1,351 & 66.1 & 216 & 10.6 & 463 & 22.6 & 14 & 0.7 & 5 & 0.2 & 2,045 & \\
\hline $5-7$ & 1,043 & 76.5 & 94 & 6.9 & 212 & 15.5 & 8 & 0.6 & 8 & 0.6 & 1,364 & \\
\hline \multicolumn{13}{|l|}{ Month } \\
\hline October & 304 & 98.7 & 1 & 0.3 & 1 & 0.3 & 1 & 0.3 & 1 & 0.3 & 308 & \multirow{7}{*}{$<0.0001$} \\
\hline November & 396 & 96.1 & 6 & 1.5 & 8 & 1.9 & 2 & 0.5 & 0 & 0 & 412 & \\
\hline December & 463 & 86.4 & 5 & 0.9 & 67 & 12.5 & 0 & 0 & 1 & 0.2 & 536 & \\
\hline January & 541 & 68.7 & 26 & 3.3 & 217 & 27.6 & 3 & 0.4 & 2 & 0.3 & 787 & \\
\hline February & 445 & 56.1 & 67 & 8.4 & 275 & 34.7 & 4 & 0.5 & 3 & 0.4 & 793 & \\
\hline March & 366 & 48.0 & 197 & 25.8 & 190 & 24.9 & 7 & 0.9 & 5 & 0.7 & 763 & \\
\hline April & 171 & 70.7 & 49 & 20.2 & 12 & 5.0 & 7 & 2.9 & 3 & 1.2 & 242 & \\
\hline \multicolumn{13}{|l|}{ Vaccination status (all ages) } \\
\hline Unvaccinated & 1,959 & 66.4 & 308 & 10.4 & 658 & 22.3 & 15 & 0.5 & 13 & 0.4 & 2,949 & \multirow{3}{*}{$<0.0001$} \\
\hline Vaccinated (14-91 days ago) & 377 & 89.8 & 6 & 1.4 & 33 & 7.9 & 3 & 0.7 & 1 & 0.2 & 420 & \\
\hline Vaccinated (>91 days ago) & 350 & 74.2 & 37 & 7.8 & 79 & 16.7 & 6 & 1.3 & 1 & 0.2 & 472 & \\
\hline Pilot area (SMN and RCGP on & & & & & & & & & & & & \\
\hline No & 1,185 & 63.8 & 181 & 9.7 & 470 & 25.3 & 20 & 1.1 & 2 & 0.1 & 1,858 & \\
\hline Yes & 91 & 72.2 & 9 & 7.1 & 24 & 19.0 & 0 & 0 & 2 & 1.6 & 126 & 0.057 \\
\hline Missing & 11 & 64.7 & 1 & 5.9 & 4 & 23.5 & 0 & 0 & 1 & 5.9 & 17 & \\
\hline Vaccine status (by route) (2- & & & & & & & & & & & & \\
\hline Not vaccinated & 402 & 65.5 & 94 & 15.5 & 112 & 18.2 & 6 & 1.0 & 1 & 0.2 & 614 & \\
\hline Injection & 16 & 84.2 & 3 & 15.8 & 0 & 0 & 0 & 0 & 0 & 0 & 19 & 001 \\
\hline Intranasal & 89 & 77.4 & 4 & 3.5 & 22 & 19.1 & 0 & 0 & 0 & 0 & 115 & 0.01 \\
\hline Missing & 12 & 70.6 & 1 & 5.9 & 4 & 23.5 & 0 & 0 & 0 & 0 & 17 & \\
\hline
\end{tabular}

RCGP: Royal College of General Practitioners Research and Surveillance Centre; SMN: Specialist Microbiology Network.

Note: Differences between cases and controls for all variables in this table were statistically significant.

${ }^{a}$ Four positive for influenza $A\left(\mathrm{H}_{1} \mathrm{~N}_{1}\right)$ and $B$; one positive for influenza $A\left(\mathrm{H}_{1} \mathrm{~N}_{1}\right)$ and $A\left(\mathrm{H}_{3} \mathrm{~N}_{2}\right)$. Duplicates are not included in row totals.

b Positive vs negative for influenza. 
Samples positive (cases; $\mathrm{n}=1,155$ ) and negative (controls; $\mathrm{n}=2,686$ ) for influenza $\mathrm{A}$ and $\mathrm{B}$ according to vaccination status and vaccine effectiveness estimates, United Kingdom, October 2015-May 2016

\begin{tabular}{|c|c|c|c|c|c|c|}
\hline & \multicolumn{2}{|c|}{ Cases } & \multicolumn{2}{|c|}{ Controls } & \multirow{2}{*}{$\begin{array}{l}\text { Crude VE } \\
(95 \% \mathrm{CI})\end{array}$} & \multirow{2}{*}{$\begin{array}{l}\text { Adjusted } \mathrm{VE} \\
(95 \% \mathrm{Cl})\end{array}$} \\
\hline & Vaccinated & Unvaccinated & Vaccinated & Unvaccinated & & \\
\hline Influenza A or B & 165 & 990 & 727 & 1,959 & $\begin{array}{c}55.1 \\
(45.9-62.7) \\
\end{array}$ & $\begin{array}{c}52.4 \\
(41.0-61.6)\end{array}$ \\
\hline Influenza $\mathrm{A}\left(\mathrm{H}_{1} \mathrm{~N}_{1}\right)$ & 112 & 658 & 727 & 1,959 & $\begin{array}{c}54.1 \\
(43-63.1) \\
\end{array}$ & $\begin{array}{c}54.5 \\
(41.6-64.5) \\
\end{array}$ \\
\hline Influenza $\mathrm{A} / 6 \mathrm{~B} 1^{\mathrm{b}}$ & 45 & 232 & 651 & 1,739 & $\begin{array}{c}48.2 \\
(28.8-62.8)\end{array}$ & $\begin{array}{c}48.9 \\
(26.4-64.5)\end{array}$ \\
\hline Influenza B & 43 & 308 & 727 & 1,959 & $\begin{array}{c}62.4 \\
(47.7-73.0) \\
\end{array}$ & $\begin{array}{c}54.2 \\
(33.1-68.6)\end{array}$ \\
\hline $\begin{array}{l}\text { Influenza B/ } \\
\text { Victoria }^{\text {b }}\end{array}$ & 21 & 161 & 651 & 1,739 & $\begin{array}{c}65.2 \\
(44.6-78.1) \\
\end{array}$ & $\begin{array}{c}57.3 \\
(28.4-74.6) \\
\end{array}$ \\
\hline
\end{tabular}

Cl: confidence interval; RCGP: Royal College of General Practitioners Research and Surveillance Centre; VE: vaccine effectiveness.

a Adjusted for age group, sex, month, pilot area and surveillance scheme.

${ }^{b}$ Based only on data from RCGP and Scotland only.

Table 3 shows that the adjusted VE was 54.5\% (95\% Cl: 41.6-64.5) against influenza $\mathrm{A}\left(\mathrm{H}_{1} \mathrm{~N}_{1}\right)$ pdmog and specifically $48.9 \%$ (95\% Cl: $26.4-$ $64.5)$ for clade $6 \mathrm{~B}_{1}$ viruses. The age-specific VE against influenza $\mathrm{A}\left(\mathrm{H}_{1} \mathrm{~N}_{1}\right)$ pdmog ranged from $48.5 \%(95 \% \mathrm{Cl}: 8.5-71.0)$ in those aged two to 17 years to $59.8 \%(95 \% \mathrm{Cl}: 35.8-74.8)$ in those aged 18 to 44 years (Table 4$)$. There was no significant difference in $\mathrm{VE}$ against influenza $\mathrm{A}(\mathrm{H} 1 \mathrm{~N} 1)$ pdmog by time since vaccination or period of vaccination (Table 4), overall or by age (adult/child).

strain. More recent substitutions observed this season included N116K, K298E, E312K and also L172Q seen in the majority of $\mathrm{B} /$ Yamagata clade 3 viruses.

Of 99 influenza B viruses isolated from sentinel sources between December 2015 and May 2016 and analysed by $\mathrm{HI}$ assay, 98 (99\%) were characterised as belonging to the $B / V i c t o r i a / 2 / 87$ lineage and were antigenically similar to B/Brisbane/60/2008, the influenza B/Victorialineage component of the $2015 / 16$ northern hemisphere quadrivalent vaccines. One virus (1\%) was characterised as belonging to the B/Yamagata/16/88-lineage and was antigenically similar to B/Phuket/3073/2013, the influenza B/Yamagata-lineage component of the 2015/16 northern hemisphere trivalent and quadrivalent vaccines.

\section{Model fitting for vaccine effectiveness estimation}

The variables incorporated in the multivariable model (month of sample collection, age group, sex, surveillance scheme and primary school programme area) were all significantly associated with swab positivity, and all except primary school programme area and sex were confounders for the vaccine effects (changed estimates by more than $5 \%$ ). As with previous seasons' analyses [5-7], risk group was not included in the final model as it was not a confounder and data were missing for 119 samples (3.1\%).

The crude and adjusted VE estimates against all confirmed influenza, influenza $A\left(\mathrm{H}_{1} \mathrm{~N}_{1}\right)$ pdmog and influenza B for the 2015/16 season are given in Table 3. There were inadequate numbers to estimate VE against influenza $\mathrm{A}\left(\mathrm{H}_{3} \mathrm{~N}_{2}\right)$. The adjusted VE was $52.4 \%(95 \%$ confidence interval $(\mathrm{Cl}):$ 41.0-61.6) against all laboratory-confirmed influenza for all ages.

Table 3 shows that the adjusted VE was 54.5\% (95\% $\mathrm{Cl}$ : 41.6-64.5) against influenza $\mathrm{A}\left(\mathrm{H}_{1} \mathrm{~N}_{1}\right)$ pdmog and specifically $48.9 \%(95 \% \mathrm{Cl}: 26.4-64.5)$ for clade $6 \mathrm{~B} 1$ viruses. The age-specific VE against influenza $A\left(\mathrm{H}_{1} \mathrm{~N}_{1}\right)$ pdmog ranged from $48.5 \%(95 \% \mathrm{Cl}: 8.5-71.0)$ in those aged two to 17 years to $59.8 \%(95 \% \mathrm{Cl}: 35.8-74.8)$ in those aged 18 to 44 years (Table 4 ). There was no significant difference in VE against influenza $A\left(\mathrm{H}_{1} \mathrm{~N}_{1}\right)$ pdmog by time since vaccination or period of vaccination (Table 4), overall or by age (adult/child).

Table 3 also shows that the adjusted VE was $54.2 \%$ (95\% Cl: 33.1-68.6) against influenza B and specifically $57.3 \%$ (95\% Cl: $28.4-74.6$ ) for viruses of the B/Victoria lineage. The age-specific VE against influenza $B$ ranged from $76.5 \%(95 \% \mathrm{Cl}: 41.9-90.5)$ in those aged two to 17 years to $-20.0 \%$ ( $95 \% \mathrm{Cl}:-259.1$ to 59.8$)$ in those aged 65 years and older (Table 4 ), although these agespecific differences in VE were not significant. There was no significant difference in influenza B VE by time since vaccination or by period of vaccination (Table 4 ).

The VE results by type of vaccine in children two to 17 years of age are given in Table 5 . For children receiving LAIV, the overall VE against all laboratory-confirmed influenza was $57.6 \%$ (95\% Cl: $25.1-76)$ and specifically 81.4\% (95\% Cl: 39.6-94.3) for influenza B and $41.5 \%$ ( $95 \% \mathrm{Cl}:-8.5$ to 68.5$)$ for influenza $A\left(\mathrm{H}_{1} \mathrm{~N}_{1}\right)$ pdmo9. This compares to an overall VE of $77.8 \%$ (95\% Cl: $7.3-94.7)$ for children receiving IIV and a specific VE of $56.3 \%$ (95\%Cl: -121.6 to 91.4 ) against influenza $\mathrm{B}$ and $100 \%$ (95\% Cl: 13.3-100) against influenza $A\left(\mathrm{H}_{1} \mathrm{~N}_{1}\right)$ pdmo9. By age group, overall LAIV VE in two to eight year-olds was 
TABLE 4

Adjusted vaccine effectiveness estimates for influenza by age, time since vaccination, vaccination period and risk group, United Kingdom, October 2015-May 2016 $(\mathrm{n}=3,841)$

\begin{tabular}{|c|c|c|c|c|}
\hline \multirow{2}{*}{ Factor } & \multirow{2}{*}{ Level } & \multicolumn{3}{|c|}{$\begin{array}{c}\text { Adjusted VEa by type } \\
\%(95 \% \mathrm{Cl})\end{array}$} \\
\hline & & $A+B$ & $\begin{array}{l}\mathrm{A}\left(\mathrm{H}_{1} \mathrm{~N}_{1}\right) \\
\mathrm{pdmo9}\end{array}$ & B \\
\hline \multirow{4}{*}{ Age (years) ${ }^{b}$} & $2-17$ & $\begin{array}{c}60.6 \\
(34.4-76.3) \\
\end{array}$ & $\begin{array}{c}48.5 \\
(8.5-71.0) \\
\end{array}$ & $\begin{array}{c}76.5 \\
(41.9-90.5) \\
\end{array}$ \\
\hline & $18-44$ & $\begin{array}{c}55 \cdot 3 \\
(34.2-69.6) \\
\end{array}$ & $\begin{array}{c}59.8 \\
(35.8-74.8) \\
\end{array}$ & $\begin{array}{c}45.9 \\
(1.0-70.4) \\
\end{array}$ \\
\hline & $45-64$ & $\begin{array}{c}55.4 \\
(34.6-69.5)\end{array}$ & $\begin{array}{c}58.6 \\
(36.9-72.8)\end{array}$ & $\begin{array}{c}65.0 \\
(15.1-85.6)\end{array}$ \\
\hline & $\geq 65$ & $\begin{array}{c}29.1 \\
(-34.1 \text { to } 61.8) \\
\end{array}$ & $\begin{array}{c}56.1 \\
(7.2-79.3) \\
\end{array}$ & $\begin{array}{c}-20.2 \\
(-259.1 \text { to } 59.8)\end{array}$ \\
\hline \multirow{2}{*}{$\begin{array}{l}\text { Period of } \\
\text { vaccination }^{b}\end{array}$} & Oct - Jan & $\begin{array}{c}50.0 \\
(27.6-65.4) \\
\end{array}$ & $\begin{array}{c}54 \cdot 3 \\
(31.6-69.4) \\
\end{array}$ & $\begin{array}{c}35.9 \\
(-70.5 \text { to } 75.9) \\
\end{array}$ \\
\hline & Feb-April & $\begin{array}{c}53.0 \\
(38.7-64.0)\end{array}$ & $\begin{array}{c}53.6 \\
(36.1-66.3)\end{array}$ & $\begin{array}{c}56.9 \\
(35.1-71.3)\end{array}$ \\
\hline \multirow{2}{*}{$\begin{array}{l}\text { Time from } \\
\text { vaccination } \\
\text { to onset }\end{array}$} & <3 months & $\begin{array}{c}51.4 \\
(29.9-66.3) \\
\end{array}$ & $\begin{array}{c}56.7 \\
(34.9-71.3) \\
\end{array}$ & $\begin{array}{c}53.1 \\
(-12.1 \text { to } 80.3) \\
\end{array}$ \\
\hline & >3 months & $\begin{array}{c}52.7 \\
(39.2-63.2)\end{array}$ & $\begin{array}{c}53.9 \\
(38.1-65.6)\end{array}$ & $\begin{array}{c}53.4 \\
(30.0-69.0)\end{array}$ \\
\hline
\end{tabular}

$\mathrm{Cl}$ : confidence interval; VE: vaccine effectiveness

a Adjusted for age group, sex, month, pilot area and surveillance scheme. ${ }^{b}$ No significant evidence of interaction.

$50.2 \%(95 \% \mathrm{Cl}: 1.6-74.8)$ and $63.9 \%(95 \% \mathrm{Cl}:-20.3$ to 89.2 ) in nine to 17 year olds.

In $2013 / 14$, the dominant circulating strain was influenza $A\left(\mathrm{H}_{1} \mathrm{~N}_{1}\right)$ pdmo9, whereas in $2014 / 15$, the dominant circulating strain was influenza $A\left(\mathrm{H}_{3} \mathrm{~N}_{2}\right)$, which had antigenically and genetically drifted from the vaccine strain, followed by influenza B mainly of the B/ Yamagata lineage. Over the three seasons, the overall VE of LAIV was $53.1 \%(95 \% \mathrm{Cl}: 31.4-67.9)$ against all confirmed influenza, with a VE of $31.5 \%(95 \% \mathrm{Cl}:-50.4-$ 68.8) for IIV (Table 6). The LAIV VE showed evidence of significant VE against laboratory-confirmed influenza $B$ infection, borderline significance against influenza $\mathrm{A}\left(\mathrm{H}_{3} \mathrm{~N}_{2}\right)$ and moderate, non-significant effectiveness against influenza $A\left(\mathrm{H}_{1} \mathrm{~N}_{1}\right)$ pdmog. Over the three-year period, albeit with small numbers, there was no evidence of significant effectiveness of IIV against influenza $B$ or $A\left(\mathrm{H}_{3} \mathrm{~N}_{2}\right)$, but effectiveness of $100 \%(95 \% \mathrm{Cl}$ : 16.2-100) against influenza $A\left(\mathrm{H}_{1} \mathrm{~N}_{1}\right)$ pdmog.

\section{Discussion}

In the 2015/16 season, the UK completed the third season of the introduction of a universal paediatric LAIV programme. The 2015/16 season was characterised by late, prolonged influenza $\mathrm{A}\left(\mathrm{H}_{1} \mathrm{~N}_{1}\right)$ pdmog activity, with predominance of an emerging genetic HA subgroup, which was antigenically well matched to the vaccine strain, followed by circulation of influenza B viruses, predominantly of the $B / V i c t o r i a$ lineage which was not represented in the 2015/16 trivalent inactivated influenza vaccine. The end-of-season VE was moderately good in adults for influenza $A\left(\mathrm{H}_{1} \mathrm{~N}_{1}\right)$ pdmog and in adultsyounger than 65 years for influenza $B$, despite the $B$ lineage mismatch for the trivalent influenza vaccine, the main vaccine used in adults. Overall VE for LAIV in children was also moderately good and specifically for influenza $B$, it was very good, although protection was less against influenza $A\left(\mathrm{H}_{1} \mathrm{~N}_{1}\right)$ pdmo9. There was no evidence to suggest waning vaccine-derived protection or changes in circulating strains over the 2015/16 season.

We found an overall significant VE of $52.4 \%$ and specifically of $54.5 \%$ against influenza $A\left(\mathrm{H}_{1} \mathrm{~N}_{1}\right)$ pdmo9, the dominant circulating strain this season. Although $2015 / 16$ has seen the continued emergence of the new genetic subgroups 6B.1 and 6B.2, the antigenic characterisation indicates a good match to the 2015/16 influenza vaccine strain and no measurable differences between these two emerging groups, which reinforces the VE findings in this paper. These levels of effectiveness are consistent with those reported mid-season in 2015/16 [5], but also in earlier $A\left(\mathrm{H}_{1} \mathrm{~N}_{1}\right)$ pdmog seasons, in particular in 2010/11 [14]. The 2015/16 A(H1N1) pdmog VE results were also similar to the mid-season estimates reported from North America and elsewhere in Europe this season $[15,16]$. The continuing apparent antigenic and epidemiological match to the vaccine strain remains encouraging and supports the World Health Organization's recommendation that the vaccine for the 2016/17 northern hemisphere winter should include an A/California/7/2009-like vaccine strain [17].

In younger adults under 65 years of age, influenza B VE was over $50 \%$. Almost all vaccinated adults in the UK can be expected to have received the 2015/16 trivalent inactivated (rather than the quadrivalent) influenza vaccine, which contained the B/Yamagata vaccine strain in 2015/16. Our results indicate that despite this lineage mismatch, the 2015/16 IIV in younger adults continued to provide important levels of protection against influenza $B$, findings which are consistent with earlier published literature [18]. On the other hand, we failed to find evidence of significant VE against influenza $B$ in the elderly, although underpowered with only 19 positive detections and a low positivity of $4.6 \%$ in this age group. This is in contrast to the $2014 / 15$ season, when influenza vaccines elsewhere in Europe provided effectiveness of $50.4 \%$ (95\% Cl: 14.6-71.2) against influenza B in thoseolder than 65 years [19]; in that season, the dominant circulating strain was $B /$ Yamagata and belonged to a clade that was antigenically similar to the vaccine virus that season. Evidence of cross-protection, as we seem to have seen in the younger adults this season, might have important implications for the potential incremental cost-effectiveness and recommendations for preferential use of quadrivalent vaccines in adults and highlights the importance of gathering further data in this area to better inform such decisions. 
TABLE 5

Vaccine effectiveness estimates for influenza by type of vaccine in two to 17 year-olds, United Kingdom, October 2015-May $2016(n=729)$

\begin{tabular}{|c|c|c|c|c|c|}
\hline Type/subtype & Type of vaccine & $\begin{array}{c}\text { Cases } \\
\text { (unvaccinated; } \\
\text { vaccinated) }\end{array}$ & $\begin{array}{c}\text { Controls } \\
\text { (unvaccinated; } \\
\text { vaccinated) }\end{array}$ & $\begin{array}{l}\text { Crude VE } \\
(95 \% \mathrm{Cl})\end{array}$ & $\begin{array}{l}\text { Adjusted VEa } \\
\quad(95 \% \mathrm{Cl})\end{array}$ \\
\hline \multirow{2}{*}{ All } & Intranasal & $212 ; 26$ & $402 ; 89$ & $\begin{array}{c}44.6 \\
(11.6-65 \cdot 3)\end{array}$ & $\begin{array}{c}57.6 \\
(25.1-76)\end{array}$ \\
\hline & Injectable & $212 ; 3$ & $402 ; 16$ & $\begin{array}{c}64.4 \\
(-23.4 \text { to } 89.8)\end{array}$ & $\begin{array}{c}77.8 \\
(7 \cdot 3-94 \cdot 7)\end{array}$ \\
\hline \multirow{2}{*}{ Influenza A/(H1N1)pdmo9 } & Intranasal & $112 ; 22$ & $402 ; 89$ & $\begin{array}{c}11.3 \\
(-47.9 \text { to } 46.8)\end{array}$ & $\begin{array}{c}41.5 \\
(-8.5 \text { to } 68.5)\end{array}$ \\
\hline & Injectable & $112 ; 0$ & $402 ; 16$ & $\begin{array}{c}100 \\
(13 \cdot 3-100)\end{array}$ & $\begin{array}{c}100 \\
(13 \cdot 3-100)^{b}\end{array}$ \\
\hline \multirow{2}{*}{ Influenza B } & Intranasal & $95 ; 4$ & $402 ; 89$ & $\begin{array}{c}81 \\
(46.9-93.2) \\
\end{array}$ & $\begin{array}{c}81.4 \\
(39.7-94.3) \\
\end{array}$ \\
\hline & Injectable & $95 ; 3$ & $402 ; 16$ & $\begin{array}{c}20.7 \\
(-177.8 \text { to } 77.3)\end{array}$ & $\begin{array}{c}56.3 \\
(-121.6 \text { to } 91.4)\end{array}$ \\
\hline
\end{tabular}

$\mathrm{Cl}$ : confidence interval; VE: vaccine effectiveness.

a Adjusted for age group, sex, month, pilot area and surveillance scheme.

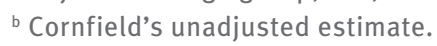

Among children two to 17 years of age, we observed an overall significant VE of $57.6 \%$ for the quadrivalent LAIV vaccine this season, specifically $81.4 \%$ for influenza $B$ and $41.5 \%$ for influenza $A\left(\mathrm{H}_{1} \mathrm{~N}_{1}\right)$ pdmog, with a similar picture when examining the previous three seasons. Over the three seasons, the overall effectiveness of LAIV was higher compared with inactivated vaccine in that age group, specifically for influenza $A\left(\mathrm{H}_{3} \mathrm{~N}_{2}\right)$ and $B$, but lower in 2015/16 and specifically for influenza $A\left(\mathrm{H}_{1} \mathrm{~N}_{1}\right)$ pdmog. These findings are in contrast to those recently reported by the US CDC who found an overall VE of only $3 \%$ for LAIV in two to 17 year-old children with very low VE against influenza $A\left(\mathrm{H}_{1} \mathrm{~N}_{1}\right)$ pdmog, while the inactivated vaccine showed significant effectiveness [3]. The US first noted lower VE of LAIV against influenza $A\left(\mathrm{H}_{1} \mathrm{~N}_{1}\right)$ pdmog in $2013 / 14$, which on further investigation was considered related to reduced thermostability of the $A / C a l i f o r n i a / 7 / 2009$ vaccine strain [20]. This led to the replacement of the $A\left(\mathrm{H}_{1} \mathrm{~N}_{1}\right)$ pdmog LAIV vaccine strain with the more recently emerged $A$ / Bolivia/559/2013 vaccine strain for the $2015 / 16$ season. Based on the 2015/16 VE findings from the CDC, the US Advisory Committee on Immunisation recommended a temporary suspension of use of LAIV for children in the US for the forthcoming 2016/17 season [3]. In addition to the UK findings presented here, Finland, in its first season of use of LAIV in pre-school age children, found overall levels of protection of $51 \%$, similar to the UK [21].

The reasons why the observed levels of overall protection were higher in Europe than in the US, with apparent reduced protection against influenza $A\left(\mathrm{H}_{1} \mathrm{~N}_{1}\right)$ pdmog compared to IIV, remain under investigation. Several hypotheses have been suggested. Firstly, are the observed differences real or the consequence of a methodological difference? If real, viral interference between the $A\left(\mathrm{H}_{1} \mathrm{~N}_{1}\right)$ pdmog vaccine strain and the other influenza vaccine viruses in the quadrivalent LAIV vaccine might provide an explanation; such interference has been discussed previously [22] and might be reinforced by prior vaccination with LAIV and/or IIV in young children (which is at present much more likely in North America than Europe) or by repeat vaccination in-season, with the US offering two doses of influenza vaccine to children compared with one dose for healthy children in Europe. A further explanation is possible antigenic drift between the A/Bolivia/559/2013 vaccine strain in the 2015/16 LAIV vaccine and circulating $\mathrm{A}\left(\mathrm{H}_{1} \mathrm{~N}_{1}\right)$ pdmog strains in winter 2015/16, although antigenically, the virus is considered to be well matched. Finally, programmatic or logistical differences, e.g. related to cold chain or vaccine handling might play a role.

Further work is required to investigate these hypotheses, although UK programme evaluation results from 2013/14 and 2014/15 already suggest that the UK LAIV paediatric programme reduced influenza circulation when comparing pilot areas where children of primary school age were offered vaccine to those areas where they were not $[23,24]$. The UK VE results presented in this paper have been reviewed by the JCVI who strongly recommended not to change the current influenza immunisation strategy planned for 2016/17, but further work is required to better understand these recent observations in the light of the US findings and to potentially optimise vaccine composition.

Although waning protection post vaccination has recently been noted [25] and although 2015/16 was a particularly late influenza season with significant activity until late into the spring, there was no evidence to suggest either waning protection by time since 
Three-season vaccine effectiveness estimates for influenza by type of vaccine in two to 17 year-olds, United Kingdom, October 2013-May $2016(n=1,655)$

\begin{tabular}{|c|c|c|c|c|c|}
\hline Type/subtype & $\begin{array}{l}\text { Type of } \\
\text { vaccine }\end{array}$ & $\begin{array}{c}\text { Cases } \\
\text { (unvaccinated; vaccinated) }\end{array}$ & $\begin{array}{c}\text { Controls } \\
\text { (unvaccinated; vaccinated) }\end{array}$ & $\begin{array}{l}\text { Crude VE } \\
(95 \% \mathrm{Cl})\end{array}$ & $\begin{array}{l}\text { Adjusted VEa } \\
\quad(95 \% \mathrm{Cl})\end{array}$ \\
\hline \multirow{2}{*}{ All } & Intranasal & $414 ; 49$ & 1,$003 ; 189$ & $\begin{array}{c}37.2 \\
(12.2-55)\end{array}$ & $\begin{array}{c}53.1 \\
(31.4-67.9)\end{array}$ \\
\hline & Injectable & $414 ; 11$ & 1,$003 ; 29$ & $\begin{array}{c}8.1 \\
(-85.7 \text { to } 54.5))\end{array}$ & $\begin{array}{c}31.5 \\
(-50.4 \text { to } 68.8)\end{array}$ \\
\hline \multirow{2}{*}{ Influenza $\mathrm{A}\left(\mathrm{H}_{3} \mathrm{~N}_{2}\right)$} & Intranasal & $129 ; 13$ & 1,$003 ; 189$ & $\begin{array}{c}46.5 \\
(3.4-70.4)\end{array}$ & $\begin{array}{c}46.7 \\
(-6.9 \text { to } 73.4)\end{array}$ \\
\hline & Injectable & $129 ; 5$ & 1,$003 ; 29$ & $\begin{array}{c}-34.1 \\
(-252.4 \text { to } 49) \\
\end{array}$ & $\begin{array}{c}-22.0 \\
(-274.8 \text { to } 60.3)\end{array}$ \\
\hline \multirow{2}{*}{ Influenza $\mathrm{A} /\left(\mathrm{H}_{1} \mathrm{~N}_{1}\right)$ pdmog } & Intranasal & $159 ; 32$ & 1,$003 ; 189$ & $\begin{array}{c}-6.8 \\
(-61 \text { to } 29.1)\end{array}$ & $\begin{array}{c}35.6 \\
(-4.4 \text { to } 60.3)\end{array}$ \\
\hline & Injectable & $159 ; 0$ & 1,$003 ; 29$ & $\begin{array}{c}100 \\
(16.2-100)\end{array}$ & $\begin{array}{c}100 \\
(16.2-100)^{b}\end{array}$ \\
\hline \multirow{2}{*}{ Influenza B } & Intranasal & $125 ; 4$ & 1,$003 ; 189$ & $\begin{array}{c}83 \\
(63.5-93.8)\end{array}$ & $\begin{array}{c}86.9 \\
(61.0-95.6)\end{array}$ \\
\hline & Injectable & $125 ; 5$ & 1,$003 ; 29$ & $\begin{array}{c}-38.3 \\
(-263.9 \text { to } 47.4)\end{array}$ & $\begin{array}{c}24.8 \\
(-153.3 \text { to } 77.7)\end{array}$ \\
\hline
\end{tabular}

$\mathrm{Cl}$ : confidence interval; VE: vaccine effectiveness.

a Adjusted for age group, sex, month, pilot area, surveillance scheme and year.

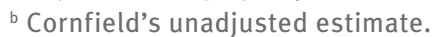

vaccination or changes in effectiveness by vaccination period due to the emergence of new clades or lineages over the course of the season in the UK. Our findings are congruent with recent work which suggests that intra-seasonal waning is of lesser importance with influenza $A\left(\mathrm{H}_{1} \mathrm{~N}_{1}\right)$ pdmog and influenza $B$ compared with influenza $A\left(\mathrm{H}_{3} \mathrm{~N}_{2}\right)$ [25].

The paper has a number of strengths. It uses a wellestablished methodology, the TNCC, the results of which approximate well to more traditional case-control approaches [26]. Data completeness was very high and the integration of genetic characterisation data has allowed the estimation of clade- and lineage-specific VE. Caution is needed in the interpretation of the results in children two to 17 years of age owing to the small sample size, particularly in relation to IIV where only a small proportion of the paediatric control population with available information (16/507, 3\%) were reported to be vaccinated, while for LAIV, $18 \%$ of controls were reported vaccinated.

\section{Conclusion}

In summary, notwithstanding the limitation of the small sample size, our findings together with those from Finland confirm encouraging overall levels of protection for LAIV. This protection is particularly effective against influenza $B$, though less against influenza $A\left(\mathrm{H}_{1} \mathrm{~N}_{1}\right) p d m o 9$, a finding which in the light of observations in the US requires further investigation.

\section{Acknowledgements}

We are grateful to the many patients of participating practices who consent to virology swabs being taken; to the practices in the participating in the surveillance schemes in England, Wales, Northern Ireland and Scotland who supplied the clinical information on their patients; to the staff of the PHE Respiratory Virus Unit, the PHE Specialist Microbiology Laboratories, Public Health Wales Molecular Diagnostics Unit, the West of Scotland Specialist Virology Centre and the Regional Virus Laboratory, Belfast who undertook analysis of specimens. We thank the staff of PHE, RCGP RSC, Public Health Wales, Public Health Agency Northern Ireland and Health Protection Scotland teams who coordinate the GP schemes, in particular Praveen SebastianPillai and Nisha Oppilamany from PHE; Richard Lewis and Ember Hilvers from PHW; Chris Nugent and Cathriona Kearns from the PHA; Catherine Frew, Alasdair MacLean, Samantha Shepherd and Celia Aitken from WoSSVC; and Diogo Marques, Robert Seremani, Naoma William, Louise Primrose-Shaw and Amanda Burridge from HPS for overseeing data collection, and Filipa Ferreira, Ana Correa, David Mullett from RCGP RSC/University of Surrey. We acknowledge the originating and submitting laboratories of the sequences from GISAID's EpiFlu Database on which some of the analyses are based (see Table 1). All submitters of data may be contacted directly via the GISAID website www.gisaid.org

\section{Conflict of interest}

MD declares lecture fees and travel grant/ conference fees from Sanofi-Pasteur MSD in 2016; SdeL declares no direct conflict of interest, however University of Surrey has received grant funding from two Innovative Medicine Initiatives programmes ADVANCE (SdeL is a work package lead) and FLUCOP. Surrey has also received grant funding from GSK to explore the feasibility of collecting European Medicine Agency listed influenza brand-specific side effects in near real time, SdeL is PI. 


\section{Authors' contributions}

RGP led the drafting; FW, CR and NA led on the statistical analysis; JE, MG and CT led on the virological analysis; all coauthors contributed epidemiological and/or virological data, contributed to the design and interpretation of the results, reviewed drafts and approved the final version.

\section{References}

1. Hakin B, Cosford P, Harvey F. The flu immunisation programme 2013/14 - extension to children. London: Department of Health; 26 Jul 2013. Available from: https://www.gov.uk/ government/uploads/system/uploads/attachment_data/ file/225360/Children_s_flu_letter_2013.pdf

2. Public Health England (PHE). Flu plan. Winter 2015/16. London: PHE; May 2016. Available from: https://www.gov. uk/government/uploads/system/uploads/attachment data/ file/526143/Flu_Plan_Winter_2015_to_2016superseded.pdf

3. Centers for Disease Control and Prevention (CDC). ACIP votes down use of LAIV for 2016-2017 flu season. Media statement. Atlanta: CDC; 22 Jun 2016. Available from: http://www.cdc.gov/ media/releases/2016/s0622-laiv-flu.html

4. Public Health England (PHE). Surveillance of influenza and other respiratory viruses in the United Kingdom: Winter 2015 to 2016. London: PHE; 17 Mar 2016. Available from: https://www. gov.uk/government/uploads/system/uploads/attachment_ data/file/526405/Flu_Annual_Report_2015_2016.pdf

5. Pebody R, Warburton F, Ellis J, Andrews N, Potts A, Cottrell $S$, et al. Effectiveness of seasonal influenza vaccine in preventing laboratory-confirmed influenza in primary care in the United Kingdom: 2015/16 mid-season results. Euro Surveill. 2016;21(13):30179. DOI: 10.2807/1560-7917. ES.2016.21.13.30179 PMID: 27074651

6. Fleming DM, Andrews NJ, Ellis JS, Bermingham A Sebastianpillai $P$, Elliot AJ, et al. Estimating influenza vaccine effectiveness using routinely collected laboratory data. J Epidemiol Community Health. 2010;64(12):1062-7. DOI: 10.1136/jech.2009.093450 PMID: 19910645

7. Andrews N, McMenamin J, Durnall H, Ellis J, Lackenby A, Robertson C, et al. Effectiveness of trivalent seasonal influenza vaccine in preventing laboratory-confirmed influenza in primary care in the United Kingdom: 2012/13 end of season results. Euro Surveill. 2014;19(27):20851. DOI: 10.2807/15607917.ES2014.19.27.20851 PMID: 25033051

8. Ellis J, Iturriza M, Allen R, Bermingham A, Brown K, Gray J, et al. Evaluation of four real-time PCR assays for detection of influenza $A\left(\mathrm{H}_{1} \mathrm{~N}_{1}\right) v$ viruses. Euro Surveill. 2009;14(22):19230. PMID: 19497254

9. Gunson R, Maclean A, Davies E, Bennett S, Miller R, Carman WF. Development of a multiplex real-time RT-PCR that allows universal detection of influenza $A$ viruses and simultaneous typing of influenza $A / \mathrm{H}_{1} \mathrm{~N}_{1} / 2009$ virus.J Virol Methods. 2010;163(2):258-61. DOI: 10.1016/j.jviromet.2009.10.006 PMID: 19854220

10. Galiano M, Agapow P-M, Thompson C, Platt S, Underwood A, Ellis J, et al. Evolutionary pathways of the pandemic influenza A ( $\left.\mathrm{H}_{1} \mathrm{~N}_{1}\right) 2009$ in the UK. PLoS One. 2011;6(8):e23779. DOI: 10.1371/journal.pone.0023779 PMID: 21887318

11. Matrosovich M, Matrosovich T, Carr J, Roberts NA, Klenk HD Overexpression of the alpha-2,6-sialyltransferase in MDCK cells increases influenza virus sensitivity to neuraminidase inhibitors.J Virol. 2003;77(15):8418-25. DOI: 10.1128/ JVI.77.15.8418-8425.2003 PMID: 12857911

12. Zambon M. Laboratory Diagnosis of Influenza. In: Nicholson K, Hay A, Webster RG, editors. Textbook of Influenza. Oxford: Blackwell Science; 1998. pp. 291-313].</edb〉

13. Tamura K, Stecher G, Peterson D, Filipski A, Kumar S. MEGA6: Molecular Evolutionary Genetics Analysis version 6.0.Mol Bio Evol. 2013;30(12):2725-9. DOI: 10.1093/molbev/mst197 PMID: 24132122

14. Pebody R, Hardelid P, Fleming D, McMenamin J, Andrews N, Robertson C, et al. Effectiveness of seasonal 2010/11 and pandemic influenza $\mathrm{A}\left(\mathrm{H}_{1} \mathrm{~N}_{1}\right)_{2009}$ vaccines in preventing influenza infection in the United Kingdom: mid-season analysis 2010/11. Euro Surveill. 2011;16(6):19791.PMID: 21329644

15. Kissling E, Valenciano M. Early influenza vaccine effectiveness results 2015-16: I-MOVE multicentre case-control study. Euro Surveill. 2016;21(6):30134. DOI: 10.2807/1560-7917. ES.2016.21.6.30134 PMID: 26898240

16. Chambers C, Skowronski D, Sabaiduc S, Winter A, Dickinson J, De Serres G, et al. Interim estimates of 2015/16 vaccine effectiveness against influenza $\mathrm{A}\left(\mathrm{H}_{1} \mathrm{~N}_{1}\right)$ pdmog, Canada, February 2016. Euro Surveill. 2016;21(11):30168.
17. World Health Organization (WHO). Recommended composition of influenza virus vaccines for use in the 2016-17 northern hemisphere influenza season. Geneva: WHO; 25 February 2016. Available from: http://www.who.int/influenza/vaccines/virus/ recommendations/2016_17_north/en

18. McLean HQ, Thompson MG, Sundaram ME, Kieke BA, Gaglani $M$, Murthy $K$, et al. Influenza vaccine effectiveness in the United States during 2012-2013: variable protection by age and virus type. J Infect Dis. 2015;211(10):1529-40. DOI: 10.1093/ infdis/jiu647 PMID: 25406334

19. Valenciano M, Kissling E, Reuss A, Rizzo C, Gherasim A, Horváth JK, et al. Vaccine effectiveness in preventing laboratory-confirmed influenza in primary care patients in a season of co-circulation of influenza $A\left(\mathrm{H}_{1} \mathrm{~N}_{1}\right)$ pdmog, $B$ and drifted $A\left(\mathrm{H}_{3} \mathrm{~N}_{2}\right)$, I-MOVE Multicentre Case-Control Study, Europe 2014/15. Euro Surveill. 2016;21(7):30139. DOI: 10.2807/1560-7917.ES.2016.21.7.30139 PMID: 26924024

20. Caspard H, Gaglani M, Clipper L, Belongia EA, McLean HQ, Griffin MR, et al. Effectiveness of live attenuated influenza vaccine and inactivated influenza vaccine in children 2-17 years of age in 2013-2014 in the United States. Vaccine. 2016;34(1):77-82. DOI: 10.1016/j.vaccine.2015.11.010 PMID: 26589519

21. Nohynek H, Baum U, Syrjänen R, Ikonen N, Sundman J, Jokinen J. Effectiveness of the live attenuated and the inactivated influenza vaccine in two-year-olds - a nationwide cohort study Finland, influenza season 2015/16. Euro Surveill. 2016;21(38):30346. DOI: 10.2807/1560-7917. ES.2016.21.38.30346

22. Bandell A, Woo J, Coelingh K. Protective efficacy of liveattenuated influenza vaccine (multivalent, Ann Arbor strain): a literature review addressing interference. Expert Rev Vaccines. 2011;10(8):1131-41. DOI: 10.1586/erv.11.73 PMID: 21854309

23. Pebody RG, Green HK, Andrews N, Boddington NL, Zhao H, Yonova I, et al. Uptake and impact of vaccinating school age children against influenza during a season with circulation of drifted influenza A and B strains, England, 2014/15. Euro Surveill. 2015;20(39):30029. DOI: 10.2807/1560-7917. ES.2015.20.39.30029 PMID: 26537222

24. Pebody RG, Green HK, Andrews N, Zhao H, Boddington N, Bawa Z, et al. Uptake and impact of a new live attenuated influenza vaccine programme in England: early results of a pilot in primary school-age children, 2013/14 influenza season. Euro Surveill. 2014;19(22):20823. DOI: 10.2807/1560-7917. ES2014.19.22.20823 PMID: 24925457

25. Kissling E, Nunes B, Robertson C, Valenciano M, Reuss A, Larrauri $A$, et al. I-MOVE multicentre case-control study $2010 / 11$ to $2014 / 15$ : Is there within-season waning of influenza type/subtype vaccine effectiveness with increasing time since vaccination? Euro Surveill. 2016;21(16):30201. DOI: 10.2807/1560-7917.ES.2016.21.16.30201 PMID: 27124420

26. Orenstein EW, De Serres G, Haber MJ, Shay DK, Bridges CB, Gargiullo $\mathrm{P}$, et al. Methodologic issues regarding the use of three observational study designs to assess influenza vaccine effectiveness. Int J Epidemiol. 2007;36(3):623-31. DOI: 10.1093/ije/dymo21 PMID: 17403908

\section{License and copyright}

This is an open-access article distributed under the terms of the Creative Commons Attribution (CC BY 4.0) Licence. You may share and adapt the material, but must give appropriate credit to the source, provide a link to the licence, and indicate if changes were made.

This article is copyright of the authors, 2016. 